Meta

Journal des traducteurs

Translators' Journal

\title{
Le rôle de l'imagerie dans la communication réalisée par un interprète
}

\section{Christian Degueldre}

Volume 47, numéro 1, mars 2002

URI : https://id.erudit.org/iderudit/007992ar

DOI : https://doi.org/10.7202/007992ar

Aller au sommaire du numéro

Éditeur(s)

Les Presses de l'Université de Montréal

ISSN

0026-0452 (imprimé)

1492-1421 (numérique)

Découvrir la revue

Citer cet article

Degueldre, C. (2002). Le rôle de l'imagerie dans la communication réalisée par un interprète. Meta, 47(1), 58-86. https://doi.org/10.7202/007992ar
Résumé de l'article

Parmi les nombreux aspects de la communication en interprétation, celui qui a été peu étudié dans la littérature et peu abordé dans la formation des interprètes dans les écoles de par le monde, mais qui attire notre attention de façon particulière, c'est l'imagerie ou la représentation mentale. La représentation mentale a été définie notamment par Shepard (1978, p. 89) qui décrit un continuum de représentations mentales qui va depuis l'image la plus concrète (et visuelle) jusqu'à des représentations non visuelles de concepts, à savoir des idées sans image. Ce processus, qui consiste à construire une image ou une représentation mentale des concepts ou idées exprimés lors de la communication, a fait l'objet de nombreuses études dans le domaine de la psychologie cognitive, notamment en ce qui concerne la communication interpersonnelle. La présente recherche examine l'importance et le rôle des représentations mentales et des unités cognitives dans le travail des interprètes au moment où ceux-ci les associent à leur mémoire à court et à moyen terme pour reproduire un message entendu et démontre qu'il pourrait être utile d'insister sur l'importance de l'imagerie et de la représentation mentale dans la formation des interprètes dans les écoles et universités.
Ce document est protégé par la loi sur le droit d'auteur. L’utilisation des services d’Érudit (y compris la reproduction) est assujettie à sa politique d'utilisation que vous pouvez consulter en ligne.

https://apropos.erudit.org/fr/usagers/politique-dutilisation/ 


\title{
Le rôle de l'imagerie dans la communication réalisée par un interprète
}

\author{
CHRISTIAN DEGUELDRE \\ Monterey Institute of International Studies, Monterey, États-Unis
}

\begin{abstract}
RÉSUMÉ
Parmi les nombreux aspects de la communication en interprétation, celui qui a été peu étudié dans la littérature et peu abordé dans la formation des interprètes dans les écoles de par le monde, mais qui attire notre attention de façon particulière, c'est l'imagerie ou la représentation mentale.

La représentation mentale a été définie notamment par Shepard $(1978$, p. 89) qui décrit un continuum de représentations mentales qui va depuis l'image la plus concrète (et visuelle) jusqu'à des représentations non visuelles de concepts, à savoir des idées sans image. Ce processus, qui consiste à construire une image ou une représentation mentale des concepts ou idées exprimés lors de la communication, a fait l'objet de nombreuses études dans le domaine de la psychologie cognitive, notamment en ce qui concerne la communication interpersonnelle.

La présente recherche examine l'importance et le rôle des représentations mentales et des unités cognitives dans le travail des interprètes au moment où ceux-ci les associent à leur mémoire à court et à moyen terme pour reproduire un message entendu et démontre qu'il pourrait être utile d'insister sur l'importance de l'imagerie et de la représentation mentale dans la formation des interprètes dans les écoles et universités.
\end{abstract}

\begin{abstract}
One of the many aspects of interpreting to which little attention has been paid both in writings on the subject and interpreter training around the world is imaging or mental representation. Defined [by Shepard $(1978$, p. 89] as a continuum of mental representations, starting with the most concrete visual image and extending to imageless thought, this process which consists of constructing mental images of concepts or ideas expressed during communication, has been widely studied in cognitive psychology, especially in interpersonal communication. This article examines the role and importance of mental representations and cognitive units and the relationship between these representations and short-term and medium-term memory during interpretation. It also demonstrates the importance of mental imaging in interpreter training in schools and universities.
\end{abstract}

\section{MOTS-CLÉS/KEYWORDS}

cognition, imagerie, interprétation, mémoire à court terme et à long terme, représentation mentale

\section{Introduction}

En tant que professeur d'interprétation et de traduction chargé de la formation d'étudiants à la maîtrise en interprétation de conférence tant à Hankuk University of Foreign Studies, à Séoul (de 1980 à 1987) qu'à la Graduate School of Translation and Interpretation du Monterey Institute of International Studies (de 1987 à ce jour), et comme interprète moi-même, je me suis intéressé aux nombreuses difficultés ren- 
contrées pour "faire passer» un message entre deux ou plusieurs personnes. J'ai donc voulu voir dans quelle mesure la communication par l'intermédiaire d'un interprète est différente par rapport à la communication monolingue.

Vu l'ampleur du domaine étudié et la multiplicité de facteurs qui affectent la communication, j'ai voulu limiter cette étude à une seule caractéristique de l'acte communicatif: la représentation visuelle ou l'imagerie mentale. Cet article a pour objectif d'aborder le travail de l'interprète sous l'angle de la psychologie cognitive et de voir comment il utilise l'imagerie et la représentation mentale au moment de la compréhension, de l'abstraction et de la ré-expression du message.

Dans quelle mesure la représentation mentale est-elle activée de manière différente selon le type de texte, de discours, ou d'intervention (concrète et abstraite) ? Les interprètes expérimentés utilisent-ils la visualisation de manière différente par rapport aux étudiants en formation? Et si oui, ne serait-il pas utile dès lors de souligner l'importance de l'imagerie-représentation dans la formation des interprètes?

Les questions qui me préoccupent essentiellement sont les suivantes:

1) voir si les conclusions auxquelles la psychologie cognitive est arrivée, notamment quant à l'importance de l'imagerie dans la compréhension (Denis, $1989^{1}$; Le $\mathrm{Ny}, 1982^{2}$ ), rétention et reproduction de messages, sont applicables à l'interprétation - les interprètes trouvent-ils également que «les idées concrètes sont plus faciles à restituer que des idées plus abstraites ${ }^{3}$ » et les images sont-elles plus faciles à restituer que les représentations plus abstraites et moins visuelles; 2) déterminer s'il existe une différence entre les interprètes chevronnés et novices quant à la manière dont chacun utilise ou non des représentations mentales en phase de reproduction; 3) les représentations formées sont-elles généralement mieux rendues par l'interprète que les passages des textes pour lesquels il n'y a pas eu de représentation mentale.

Toutes ces questions ont, pour moi, un objectif primordial qui est l'application pédagogique des résultats obtenus: peut-on apprendre à représenter des idées abstraites aussi bien que des idées concrètes grâce à un travail conscient d'imagerie et de représentation.

Ne pouvant évidemment répondre à l'ensemble de ces questions en une seule étude, j'ai dû me limiter à une seule question: l'imagerie est-elle utile dans le travail de l'interprète? Pour répondre à cette question, j'ai conçu un instrument de recherche (voir la deuxième partie) qui je présenterai après une brève révision de la littérature.

\section{PREMIËRE PARTIE : RÉVISION DE LA LITTÉRATURE}

\section{Place de la représentation mentale et de l'imagerie dans la communication et la compréhension}

Dans de nombreux programmes de formation d'interprètes, l'approche très pragmatique de l'enseignement ne permet pas de sensibiliser les étudiants suffisamment à ses aspects plus théoriques. Ainsi peu de travaux ont jusqu'à présent été consacrés à l'apport de l'imagerie et de la résolution de problèmes. Moins encore se penchent sur le rôle de l'imagerie dans le travail de l'interprète 4 . Les travaux existant [Kaufmann (1985) ; Kosslyn (1983, chap. 10); Richardson (1983)] traitent plutôt de l'imagerie et de problèmes de logique spatiale. 
Aussi, pour aider le lecteur à situer l'imagerie et la représentation mentale appliquées à l'interprète, je voudrais commencer par un très succinct rappel des principes généraux de la communication et de la compréhension. Ce faisant, je suis conscient que je laisserai évidemment de côté de nombreux éléments de la compréhension et de la communication.

\section{LA COMMUNICATION}

La communication parfaite est illusoire (Sperber, 1986), l'expérience de notre vie quotidienne nous le rappelle trop souvent. Ainsi, Sperber nous rappelle que les idées ne peuvent être transportées d'une tête à l'autre et que même le meilleur écrivain ne peut être sûr d'avoir réussi complètement à faire comprendre son message exactement de la même manière qu'il l'aurait souhaité. Des messages simples peuvent nous donner l'illusion d'une communication parfaite. Dans certains cas, il est permis de se rendre compte si la communication est "passée» en vérifiant la compréhension du message (par exemple: la compréhension du message «Passez-moi le livre qui se trouve sur la table» sera vérifiée si la personne à qui il est destiné apporte le livre demandé).

Néanmoins, la communication reste un processus complexe qui fait intervenir un grand nombre de facteurs (écoute, attention, concentration, mémoire, fatigue, connaissance du sujet, contexte, capacité d'inférer, abstraction du sens, reformulation... entre autres).

\section{Modèles de communication}

La communication (verbale ou autre) va bien au-delà de la simple expression de nos pensées à l'aide de mots (le codage et décodage — «nos pensées, elles, restent confinées à notre cerveau») $)^{5}$. Ray Jackendoff (1993) parle d'un processus consistant à passer du niveau des idées à des instructions motrices (c'est la production orale), complété par la perception auditive de l'auditeur qui lui permet de reconstituer ces idées. Ainsi, selon lui, les différentes langues seraient des manières différentes de transformer les pensées, les idées en schémas moteurs (motor patterns) et les perceptions auditives en idées.

Sperber et Wilson (1986) introduisent la notion de communication ostensiveinférentielle (Ostensive-inferential communication) qui se fonde sur des suppositions (Assumptions). Outre la référence obligatoire au code (dans le cas qui nous occupe un code linguistique, c'est-à-dire la capacité de reconnaître et d'utiliser les signes d'une langue), la communication implique la volonté de communiquer et la capacité de créer des inférences de la part du locuteur et la capacité de percevoir ces inférences de la part de son interlocuteur.

Le modèle de communication présenté par Shannon et Weaver (1949) faisait intervenir le codage et le décodage linguistique ainsi que des central thought processes (processus mentaux centraux). C'est à ce niveau que se fait la représentation de l'information. En effet, il y a un écart entre les représentations sémantiques des phrases et les pensées exprimées par la parole. Cet écart est comblé, selon Sperber et Wilson, non pas par le codage mais par les inférences... La communication peut donc être décrite comme étant un processus de reconnaissance par inférence des intentions de 
la personne qui communique. Lorsque nous créons des inférences, nous essayons de connecter des éléments nouveaux d'information à des éléments stockés dans notre mémoire (nous verrons par la suite l'importance de la mémoire chez l'interprète).

\section{Communication monolingue et bilingue}

La communication entre personnes partageant une même langue (code), une même culture et des réalités/expériences similaires est déjà difficile, car elle dépend aussi de la faculté d'établir des inférences et de les vérifier, ainsi que de la connaissance que chacune d'entre elles a du monde.

La communication par le biais d'un interprète ou d'un traducteur (Bell, 1991) représente un véritable défi.

En effet, l'interprète est à la fois RÉCEPTEUR et ÉMETTEUR. En tant que récepteur, il utilise, entre autres outils à sa disposition, sa capacité de représentation (son imagerie mentale) pour traiter des informations et résoudre les problèmes qu'elles posent sur le plan de la compréhension. Émetteur, il devra reconstituer le message sur la base de sa compréhension de l'ensemble de départ. Il aura pour l'aider sa mémoire, sa culture, ses connaissances, son expérience, l'analyse qu'il aura faite du message, et les représentations et images qu'il aura formées au cours de la compréhension.

La communication avec traducteur ou interprète fait intervenir deux langues, deux cultures (parfois trois, car on ne peut considérer dans tous les cas que l'interprète partage automatiquement la culture d'un des interlocuteurs), et des réalités différentes pour les trois acteurs de la communication. Elle implique qu'il faut doubler le nombre d'activités. Ainsi, en doublant le nombre de codes, d'opérations d'encodage et de décodage, en ajoutant à l'ostension une ré-ostension, en multipliant les suppositions et les inférences, on comprend aisément la quasi-impossibilité d'obtenir une équivalence à $100 \%$ entre le message dans la langue de départ et langue d'arrivée. La communication bilingue multiplie donc les risques d'incompréhension et de perte/modification d'informations; elle n'est donc jamais parfaite (Sperber et Wilson, 1986).

\section{LA COMPRÉHENSION}

\section{Qu'est-ce que comprendre?}

L'accent a souvent été mis sur l'aspect cognitif de l'interprétation. «Interpréter, c'est comprendre» (D. Seleskovitch, 1984). Mais est-ce que nous expliquons suffisamment à nos étudiants en interprétation en quoi consiste réellement la compréhension: comprennent-ils vraiment ce qu'est que comprendre?

Gérard Sabah (1993) fait à cet égard, entre autres, deux remarques intéressantes: «a) la compréhension est un réflexe (on ne peut s'empêcher de comprendre); b) l'être humain est naturellement paresseux...» et pour comprendre à un niveau donné, il est à l'affût d'informations venant de niveaux inférieurs (qui lui fournissent ses entrées). Il cherche dès lors le niveau de compréhension le plus accessible.

Que se passe-t-il lorsque nous entendons un message? Nous devons en premier lieu identifier le code utilisé; ensuite mettre en œuvre nos compétences linguistiques 
et extra-linguistiques, et activer notre capacité de créer des inférences pour comprendre. Mais comment s'assurer que ce que nous comprenons est exactement ce que le locuteur a voulu exprimer, rien de plus rien de moins et que nous ne nous contentons pas d'un niveau de compréhension trop superficiel? Comment peut-on améliorer la compréhension?

La compréhension, nous le disions, est un processus complexe qui fait intervenir un grand nombre de facteurs inter alia:

l'écoute attentive (combien d'erreurs pourraient être évitées en interprétation grâce à une meilleure écoute de la part de l'interprète),

le partage du code (la langue du message),

la capacité d'établir des inférences équivalentes du locuteur et de son interlocuteur,

la mise en contexte, et

la capacité d'utiliser les ressources de la mémoire à court et à long terme.

\section{Le partage du code}

Selon Saussure (1974:16) cité dans Sperber et Wilson (1986:7), le langage est «un système de signes qui expriment des idées et est à ce titre comparable à l'écriture, à l'alphabet des sourds-muets, aux rites symboliques, aux formules de politesse, à la signalisation militaire». Que l'on ajoute à cette liste le code Morse, la signalisation routière, le vol des abeilles... on voit dans tous les cas que pour communiquer, il faut comprendre non seulement le code sous tous ses aspects mais aussi les règles grammaticales et les règles d'usage qui le régissent. Nous savons aussi que comprendre la partie linguistique du code n'est pas tout: il faut aussi être à même de saisir les éléments non linguistiques de la communication - les gestes et expressions corporelles propres à la culture.

Pour comprendre, et partant pour communiquer, les locuteurs et leurs interlocuteurs doivent partager et respecter les mêmes normes (d'expression) de façon systématique.

\section{Les inférences}

À quoi servirait un code sans expérience passée à laquelle rattacher des éléments nouveaux, sans mémoire d'événements similaires, sans capacité d'analyse du message? La communication serait-elle possible si le locuteur et son interlocuteur n'étaient pas capables de mettre en œuvre des règles d'inférence communes sur la base de principes communs acceptés (par exemple, voler est un crime; plus est en haut)?

Normalement, lorsque les locuteurs respectent les normes d'usage (les règles d'interprétation sémantique, grammaticales, sociales et autres) et pour autant que leurs interlocuteurs s'attendent à ce qu'il en soit ainsi, le champ des interprétations possibles d'un message donné peut se réduire considérablement parce que locuteur et interlocuteur utilisent des références similaires.

"Comprendre» le message implique que le locuteur et son interlocuteur interprètent les inférences de la même manière. On peut rêver! Et surtout, on peut aussi se poser la question: comprend-on vraiment complètement? Pour élaborer des inférences, nous devons pouvoir compter sur des caractéristiques de comportements communes à des individus d'un même groupe social (Cordier, 1993). 


\section{Le contexte}

Le contexte est essentiel à la compréhension. Selon Sperber et Wilson, le contexte est «l'ensemble des conditions de base permettant d'interpréter un message [...]». Peuton toutefois supposer que les membres d'une même communauté ont la possibilité de comprendre un message donné de façon semblable (tout simplement parce qu'ils partagent une même langue et sont exposés à des événements semblables)? Rien n'est moins sûr. Ainsi, outre l'analyse de la partie linguistique d'un message (verbal ou écrit), de nombreux éléments non linguistiques interviennent comme, par exemple, l'identité du locuteur, ses intentions, les circonstances dans lesquelles s'inscrit son discours, etc. qui peuvent entraver notre compréhension. L'interprète sait à quel point il doit pouvoir placer le message dans un contexte et à quel point ses connaissances antérieures et sa préparation du sujet l'aideront à donner un sens au message.

\section{Limites de la compréhension}

Vu les difficultés mentionnées ci-dessus, est-il vraiment possible de comprendre exactement la même chose lorsque notre perception du monde et notre compréhension des messages qui nous en vient dépendent de notre connaissance du code, du contexte, de nos connaissances préalables, de notre capacité de reconnaître et d'acquérir de nouvelles connaissances par inférence, de notre mémoire, ou lorsque nous élaborons des représentations mentales de notre environnement de façons différentes? La tâche semble déjà impossible lorsque les deux interlocuteurs parlent la même langue. Elle l'est doublement lorsqu'elle fait intervenir un interprète et les risques de perte d'information n'en sont que plus grands.

\section{LA REPRÉSENTATION}

\section{Place de la représentation mentale et de l'imagerie dans la compréhension de l'interprète}

Après avoir établi que la communication parfaite est illusoire (Sperber, 1986) et que, nous venons de le voir, la compréhension parfaite semble l'être tout autant, nous allons porter notre attention sur un des outils disponibles pour améliorer la compréhension: la représentation et le rôle qu'elle joue au moment de la compréhension.

Le code (linguistique) nous permet d'associer des sons aux pensées exprimées par le locuteur. Pourquoi ne pas y associer des images ou des représentations mentales?

Du monde physique dans lequel nous vivons, nous tirons des informations sur la base desquelles nous construisons un environnement cognitif qui nous est propre et qui dépend de notre capacité de représentation, de notre mémoire, de notre vécu.

De la même manière que nous créons un environnement perceptuel (visuel, auditif, etc.) du monde qui nous entoure (et que nous voyons avec des yeux différents), nous élaborons un environnement cognitif qui nous est propre en communiquant. Pour comprendre, nous élaborons des inférences en tenant compte de suppositions de départ et nous nous rapportons à notre environnement cognitif.

La compréhension ferait donc intervenir une association entre l'image et le codage verbal: c'est le double codage, notion encore mal comprise, car on ne voit pas encore bien quel est le rôle respectif de chacun de ces deux éléments (Denis, 1993). 


\section{Qu'est-ce qu'une représentation?}

Notre compréhension du monde extérieur, et donc des messages qui nous en viennent, passe par deux voies: les sens et la représentation. Michel Denis (1986) explique que: "dans une conception multimodale de la signification, l'image peut être conçue comme l'une des composantes de la signification [...] et [elle joue un] rôle d'autant plus important que la phrase réfère à des entités matérielles susceptibles d'être figurées dans une image.»

Michel Denis (1989) nous rappelle qu' «en psychologie, le terme de 'représentation' est utilisé pour désigner à la fois un processus et le produit de ce processus. Ainsi, nous pouvons évoquer d'une part sous forme d'une image visuelle, l'objet tour Eiffel et d'autre part l'entité cognitive disponible correspondant à l'objet tour Eiffel, et qui se trouve inscrite de façon permanente dans nos structures mentales, même lorsque la tour Eiffel est totalement étrangère à notre activité mentale actuelle. On retrouve ici la distinction entre «représentations-types» (ce sont les représentations sociales de Cordier) et «représentations-occurrences» (Le Ny, 1985). « Les premières correspondent à l'information dont dispose une personne à propos d'un objet donné; les secondes, aux actualisations particulières de cette information...»

\section{Caractéristiques des représentations}

\section{La conservation et la réduction}

Entre une idée, un objet et sa représentation doit s'établir une correspondance dont un des aspects est la conservation des éléments faisant l'objet de la représentation. Vu leur degré élevé d'analogie avec l'ensemble du départ, les représentations peuvent être utilisées comme substituts partiels des objets originaux. Ainsi une photo rappelle des émotions et des souvenirs. Néanmoins, cette notion est contrebalancée par une autre notion qu'est la réduction, c'est-à-dire la perte informationnelle entre l'ensemble de départ et l'ensemble d'arrivée (la représentation). Une entité n'est porteuse d'une valeur représentationnelle que dans la mesure où est établie une relation de référence avec une entité existant en dehors d'elle. «Toute représentation est représentation de quelque chose» (Le Ny, 1985).

Pour Ida Kurcz (1993), «la représentation est souvent traitée comme étant un état ou un processus mental faisant référence à ce qui est représenté. La nature symbolique des représentations est soulignée. En d'autres termes, les représentations ne sont pas des copies ou des reflets de la réalité. Leur fonction est d'amasser des connaissances [relatives à l'objet représenté] et de s'en servir ${ }^{6}$.»

C'est aussi ce que rappelle R. Shepard (1978): 1) Les processus impliqués lorsque le cerveau élabore une image mentale ne sont pas des images mais ils doivent contenir l'information nécessaire pour, en principe, pouvoir reconstruire une image avec un isomorphisme élevé par rapport à l'objet externe imaginé ; 2) le fait que l'on a tendance normalement à penser à une image mentale sous la forme d'une image/ photo est peut-être dû au fait que le cerveau traite une image mentale pure d'une manière très similaire au traitement qu'il met en œuvre lorsqu'il regarde la photo correspondante (Shepard, 1975; Shepard et Chipman, 1970; Smart, 1959).

Posséder une représentation n'équivaut donc vraiment jamais exactement à posséder l'objet lui-même (Denis, 1985). Cette affirmation explique un des risques du 
recours qu'un interprète qui, lorsqu'il restitue un message, est constamment obligé de recréer l'information sur la base des représentations qu'il a élaborées lors de l'écoute du message original. Certes, il pourra pour cela compter également sur son analyse du message, sa mémoire et sa capacité de créer des inférences et, en interprétation consécutive, sur sa prise de notes - autre forme de représentation visuelle. Le risque n'en est pas moins réel.

\section{Caractère directionnel}

On peut créer une représentation à partir d'un ensemble de départ, mais il ne sera pas possible de reconstruire l'ensemble de départ si on n'en possède que la représentation. Ceci représente une difficulté particulière pour l'interprète qui recrée le message sur la base de sa compréhension et des représentations qu'il a élaborées lors de l'écoute de la version originale.

\section{Concurrence avec les autres modes de perception}

Les expériences de Marks (1973) en matière de représentation spatiale montrent également l'importance de l'image dans le processus de compréhension. Ceci est notamment le cas lorsqu'il démontre que la perception imagée est moins affectée par une source de distraction visuelle que par une source de distraction sonore... les deux étant en concurrence pour analyser les messages. Ceci a une implication importante pour l'interprète, à qui il convient de limiter toutes distractions, surtout auditives.

\section{Utilité des représentations}

Nous analysons tout message en fonction de son utilité. Michel Denis explique que «l'efficacité (la pertinence ou l'utilité) d'une forme de représentation est étroitement dépendante des usages que l'individu est susceptible de faire de cette représentation. » Il y a sélection et activation de celles des représentations qui sont pertinentes dans un contexte "situationnel» donné (Denis). Lorsque nous comprenons un message nouveau (lors de l'analyse), nous en jugeons et jaugeons l'utilité de façon constante. Nous en déterminons la pertinence dans le contexte, nous en vérifions la "plausibilité" et nous nous en servons pour anticiper la suite du discours.

\section{Principe de la manipulation}

«Il n'y a pas de représentation sans manipulation, donc sans transformation. Les représentations ne sont généralement pas créées pour constituer, au sein du système qui les accueille, des copies dormantes du réel. Les représentations n'ont d'existence que dans la mesure où elles sont effectivement utilisées et où leur valeur sémantique est réellement exploitée. Ces aspects sont spécialement mis en relief dans les situations où les individus créent mentalement des états virtuels, non encore réalisés, du monde, en vue d'anticiper des événements ou de visualiser des hypothèses. » (Denis, 1993)

\section{Images conceptuelles et images perceptuelles}

Les images qui nous sont fournies par nos sens (images perceptuelles), si on les compare aux images que nous avons conservées dans notre mémoire d'événements vécus ou que notre imagination a créées (images conceptuelles) (sauf celles provenant de 
rêves ou d'hallucinations) sont en général beaucoup plus vives, présentes, riches en détails, en texture et en couleurs (Shepar, 1978). Elles devraient dès lors permettre une meilleure reconstruction du message.

\section{Représentation — imagerie, compréhension et mémoire}

Pour comprendre une phrase, un texte ou un discours, un sujet doit en former une représentation sémantique sur la base de l'information qui lui est fournie. L'interprète, lorsqu'il reproduit en consécutive le message entendu, doit en effectuer une deuxième lecture mentale de la représentation qu'il avait élaborée lors de la première écoute. Pour cela il utilise sa mémoire sémantique.

\section{Mémoire sémantique et mémoire cognitive}

Stéphane Ehrlich (1982) nous rappelle que: "nous avons une connaissance du monde extérieur, des objets, des événements inscrite dans la mémoire sous la forme de concepts et/ou d'images mentales (lorsque ces informations nous parviennent par le biais de nos sens). Nous parlons de mémoire sémantique lorsque ces concepts peuvent être rendus verbalement de façon explicite; sinon ils appartiennent à la mémoire cognitive générale qui comprend la mémoire sémantique.»

Ehrlich poursuit en expliquant que « chaque concept a un contenu informatif et une fonction relationnelle qui fait qu'il peut être associé à d'autres concepts pour former des éléments d'un message plus complexe.» Pour comprendre, nous devons donc connaître la valeur informative des concepts et leur valeur relationnelle. Au moment de la compréhension, nous activons dans notre mémoire à court terme (grâce aux inférences que nous formons) des éléments de la mémoire à long terme et nous établissons des liens entre l'idée nouvelle que nous découvrons et l'ensemble des connaissances accumulées vers lesquelles nous jetons des ponts pour comprendre.

La compréhension d'un interprète exige qu'il puisse disposer des mêmes informations à propos des unités sémantiques, qu'il comprenne les relations qui unissent ces unités dans un message et qu'il soit capable d'utiliser sa mémoire cognitive (ses connaissances) de manière aussi efficace que la personne dont il interprète les propos afin d'établir les mêmes ponts entre mémoire cognitive et mémoire sémantique. On prend conscience une fois encore des dangers du recours à l'interprétation.

\section{DEUXIÈME PARTIE: RECHERCHE}

\section{HYPOTHÈSE DE RECHERCHE}

Après cette brève présentation de l'importance de la représentation mentale dans la compréhension et la restitution du message et du rôle des unités cognitives dans la mémoire, il serait utile de voir comment un interprète utilise l'imagerie dans son travail et de décider s'il ne serait pas bénéfique d'insister sur la représentation mentale de façon plus systématique dans la formation des interprètes. L'hypothèse à la base de la présente recherche est que l'imagerie est utile à l'interprète. Nous souhaitons mon- 
trer qu'effectivement, la représentation mentale et l'imagerie sont des outils précieux pour les interprètes et pour cela nous allons voir: 1) quel est l'impact de l'imagerie sur la qualité du travail de l'interprète (sur sa compréhension et sa ré-expression);2) quel est l'effet des divers types de textes (concrets/abstraits) sur la restitution des messages; 3) si un interprète expérimenté a davantage recours à la représentation mentale par rapport à un novice.

En d'autres termes, les textes contenant beaucoup d'images sont-ils perçus comme étant plus faciles à restituer que les textes plus abstraits? Les textes concrets sont-ils mieux restitués, malgré leur longueur relative? L'expérience des interprètes a-t-elle un impact sur la qualité de la restitution?

\section{MÉTHODE}

Pour vérifier l'hypothèse de base de l'utilité de l'imagerie, nous nous proposons d'analyser la qualité et la quantité d'images formées par des interprètes de niveaux d'expérience divers, en fonction des différents types de textes, plus ou moins concrets. Pour cela, nous utiliserons:

1) un instrument de recherche qui comprend a) sept passages enregistrés que les répondants doivent reproduire au mieux, dans la même langue (anglais-anglais) après les avoir écouté une seule fois et b) un bref questionnaire administré à la fin de chacun des sept passages, suivi d'un questionnaire plus général administré à la fin de l'exercice (voir l'appendice 4). Les questions posées ont pour but de montrer si les passages les plus faciles à reproduire sont aussi ceux qui contiennent le plus de possibilités de représentations mentales et sont les plus concrets; et

2) des transcriptions des reproductions de chacun des répondants des deux passages qui auront été classés par la majorité comme étant respectivement le plus concret et le plus abstrait. Ces transcriptions ont pour but de voir s'il existe une corrélation entre la qualité de la restitution et le type de texte - concret ou abstrait.

\section{A. Sujets}

L'instrument final a été administré à 26 sujets regroupés pour les besoins de l'étude en trois catégories: les novices complets (9), les débutants (10) et les chevronnés (7). Au départ, nous pensions pouvoir faire une comparaison entre interprètes novices et interprètes expérimentés, mais il a fallu se rendre compte qu'il y avait dans l'échantillon un niveau intermédiaire de faux débutants, c'est-à-dire de personnes qui interprétaient de façon occasionnelle et qui souvent avaient une solide expérience en tant que traducteurs. Comme nous ne voulions pas les exclure, une catégorie supplémentaire a été créée: les débutants.

Définition des groupes: 1) les novices complets sont des étudiants dans un programme de maîtrise aux États-Unis; ces personnes entament leur formation et déclarent n'avoir qu'une expérience très limitée tant en interprétation qu'en traduction; 2) les débutants sont (a) des traducteurs, généralement membres de l'American Translators Association (ATA) qui ne travaillent comme interprètes que de façon occasionnelle, (b) des personnes qui, quoique n'ayant pas reçu de formation, ont néanmoins une bonne expérience en tant que traducteurs et qui interprètent rarement ou encore (c) des personnes qui sont à la fin de leur formation dans un programme 
d'interprétation au Canada mais qui ont moins d'un an d'expérience dans ce domaine; 3) les «chevronnés» sont des interprètes professionnels à qui l'instrument fut présenté à l'occasion de conférences internationales au niveau présidentiel et ministériel ayant eu lieu au Canada, aux Etats-Unis et au Mexique, ce qui implique au départ qu'ils avaient été sélectionnés par l'organisateur de ces conférences en fonction de leur expérience. Ils n'ont pas tous reçu une formation en interprétation mais disposent d'une longue expérience dans la pratique de l'interprétation. Pour aucun des groupes, n'ai-je voulu tenir compte de la distribution d'âge et de genre, ces caractéristiques ne me paraissant pas pertinentes.

\section{B. Description et présentation de l'instrument}

L'instrument de recherche utilisé comporte un total de 7 passages de courte durée variant entre 22 secondes (54 mots) et 1:25 minute (242 mots) (il ne fallait pas que l'exercice devienne un exercice de mémoire). Tous les extraits sont en anglais. Cinq des sept textes sélectionnés ont été enregistrés à une vitesse normale par un acteur de théâtre américain qui s'est efforcé de leur donner une intonation correcte et naturelle; les deux autres sont des extraits originaux de Hilary Clinton et de Bruce Böglin, un interprète britannique interviewé à la $\mathrm{BBC}$. Tous les extraits sont présentés à une vitesse normale également. La manière dont les textes sont présentés devait permettre de contrôler les variables d'accent et de vitesse qui auraient pu favoriser les interprètes plus expérimentés. Tous les textes ont été choisis en fonction de certaines caractéristiques propres (niveau d'abstraction, difficulté lexicale et syntaxique, familiarité avec le sujet, longueur — cf. l'appendice 1 pour plus de détails) de telle sorte que seules devaient varier la longueur et le niveau d'abstraction des extraits, notre objectif étant de voir si les textes concrets étaient mieux restitués que les textes plus abstraits, quelle que soit la longueur des passages.

\section{ÉTUDES PILOTES}

Un instrument pilote a été administré à huit étudiants en interprétation se situant à des niveaux différents de leur formation ( 5 personnes n'avaient reçu aucune formation et les 3 autres avaient terminé deux années de maîtrise dans un programme de maîtrise en interprétation aux États-Unis). Cette étude pilote a permis de modifier l'instrument utilisé afin d'assurer une plus vaste gamme de textes allant de passages concrets (par ex. Archevêque et Faire le plein d'essence) à des passages plus abstraits (Économie russe, Bhoutan) et d'éliminer des passages qui étaient trop semblables pour les remplacer par des extraits plus variés. Plusieurs modifications ont été introduites également quant à la manière dont les textes étaient présentés afin de mieux contrôler les variables de présentation de l'instrument (vitesse, accent). Ainsi, au lieu d'être lus par l'administrateur de l'instrument, les passages pour lesquels il n'y avait pas d'originaux enregistrés l'ont été par un acteur professionnel.

Cette première étude pilote a été suivie d'une deuxième administrée à quatre personnes dont le but était de s'assurer que les modifications apportées allaient bien dans le sens recherché. Un des passages remplacés (un extrait du discours de Elie Wiesel dans le cadre de la conférence du Millénaire à la Maison-Blanche) consistait essentiellement en une idée principale répétée et accompagnée d'une liste de conflits, 
de catastrophes, de guerres et de noms géographiques, ce qui ramenait l'exercice à un gigantesque effort de mémoire, et l'interprétation au niveau des mots plutôt qu'à celui des concepts qui nous intéresse.

\section{INSTRUMENT FINAL (voir l'appendice)}

\section{Conditions générales}

Les participants devaient écouter les sept passages retenus l'un à la suite de l'autre à l'aide d'écouteurs de façon à s'assurer que le son était de bonne qualité (contrôle d'une des variables de présentation). Lorsque l'instrument était utilisé dans les programmes d'interprétation aux États-Unis et au Canada, les participants se trouvaient chacun dans une cabine d'interprétation insonorisée. Dans le cas des membres de l'ATA et des interprètes à qui l'étude a été présentée lors d'une conférence, chaque répondant écoutait la cassette à l'aide d'un magnétophone haute-fidélité, dans un endroit calme. Les instructions données demandaient à chacun un maximum de concentration. En effet, l'attention joue un rôle considérable; c'est pourquoi le test n'a pas été administré à des personnes qui avouaient être fatiguées.

Chaque extrait ne peut être écouté qu'une seule fois. Les participants devaient suivre la séquence des extraits telle qu'ils étaient enregistrés sur la cassette. Ils ne pouvaient interrompre le test et le reprendre par la suite. Aucune contrainte de temps n'était imposée pour la restitution. Les participants devaient écouter chaque passage sans prendre de notes (afin de ne pas favoriser les consécutivistes expérimentés) et le reproduire dans la même langue (pour éviter les difficultés de traduction et ne pas favoriser les personnes plus expérimentées) en s'efforçant d'inclure un maximum de détails. Aucune mention n'était faite dans les consignes données du but poursuivi, étant donné que la littérature indique des résultats différents lorsque la fonction de représentation mentale est activée ${ }^{7}$ (le phénomène du désir de plaire au chercheur). Certains éléments ont été introduits pour détourner les participants de l'objectif principal du test afin qu'ils ne se concentrent pas sur cet objectif (par exemple, les sujets devaient indiquer quel avait été le terme le plus difficile dans chaque passage). Chaque restitution était enregistrée pour être analysée. Avant de passer à l'écoute du passage suivant, chaque participant devait répondre à plusieurs questions pour voir quelles avaient été, selon lui, les principales difficultés de compréhension, lexicales et autres, rencontrées. À la fin des sept extraits, chaque répondant devait remplir un formulaire d'informations personnelles pour déterminer son niveau d'expérience et de (auto-) formation et comparer les divers extraits les uns par rapport aux autres. Les répondants devaient dire dans quelle mesure les images formées avaient été utiles.

Dans la plupart des cas, l'administration du test a été suivie d'un bref entretien avec le répondant.

\section{Analyse}

Toutes les restitutions des 26 répondants ont été enregistrées et transcrites intégralement. Ensuite, les transcriptions des reproductions étaient analysées sur le plan qualitatif afin de voir s'il y avait une corrélation entre la représentation mentale et la qualité de la restitution. Un autre aspect de l'analyse provenait des réponses aux 
questionnaires données par les participants : ils devaient comparer les divers extraits les uns aux autres (cf. l'appendice 4) afin de déterminer s'il y avait des différences marquées entre les groupes.

\section{Résultats}

\section{COMPARAISON DES TEXTES}

Alors que les novices indiquaient comme texte le plus difficile celui portant sur l'économie russe, qui était pourtant court mais abstrait, suivi des autres textes abstraits sur l'économie de l'Asie du Sud-Est et sur les Pyrénées, les débutants semblaient avoir plus de difficultés avec l'extrait sur les Pyrénées, le texte plus abstrait sur l'Asie du Sud-Est et le texte sur le Bhoutan, beaucoup plus long. Certains interprètes chevronnés, habitués à la simultanée et au recours à la mémoire à court terme, ont reconnu avoir eu de sérieuses difficultés avec la longueur de l'extrait sur le Bhoutan mais ne l'ont pas indiqué comme étant le plus difficile à reproduire. Il est intéressant de remarquer que le passage qui est le plus difficile pour les novices (Texte 4 relativement abstrait) ne l'est pour aucun des interprètes chevronnés ou des débutants. Les textes les plus concrets, 3 et 6 , n'étaient pas considérés comme difficiles par aucun des groupes. Peut-on conclure que l'abstraction d'un texte présente davantage un problème pour les novices et qu'elle devient moins importante au fur et à mesure que les interprètes acquièrent de l'expérience?

TABLEAU 1

Texte le plus difficile

\begin{tabular}{|l|c|c|c|c|c|c|c|}
\hline & $\begin{array}{c}\text { Pyrénées } \\
\text { (texte 1) }\end{array}$ & $\begin{array}{c}\text { H. Clinton } \\
\text { (texte 2) }\end{array}$ & $\begin{array}{c}\text { Archbishop } \\
\text { (texte 3) }\end{array}$ & $\begin{array}{c}\text { Russian Economy } \\
\text { (texte 4) }\end{array}$ & $\begin{array}{c}\text { S-E Asia } \\
\text { (texte 5) }\end{array}$ & $\begin{array}{c}\text { Gassing up } \\
\text { (texte 6) }\end{array}$ & $\begin{array}{c}\text { Bhutan } \\
\text { (texte 7) }\end{array}$ \\
\hline Novices & 2 & & & 4 & 2 & & 1 \\
\hline Débutants & 4 & & & & 3 & & 3 \\
\hline Chevronnés $^{8}$ & 2 & & & & 2 & & 1 \\
\hline
\end{tabular}

TABleau 2

Texte le plus facile

\begin{tabular}{|l|c|c|c|c|c|c|c|}
\hline & $\begin{array}{c}\text { Pyrénées } \\
\text { (texte 1) }\end{array}$ & $\begin{array}{c}\text { H. Clinton } \\
\text { (texte 2) }\end{array}$ & $\begin{array}{c}\text { Archbishop } \\
\text { (texte 3) }\end{array}$ & $\begin{array}{c}\text { Russian Economy } \\
\text { (texte 4) }\end{array}$ & $\begin{array}{c}\text { S-E Asia } \\
\text { (texte 5) }\end{array}$ & $\begin{array}{c}\text { Gassing up } \\
\text { (texte 6) }\end{array}$ & $\begin{array}{c}\text { Bhutan } \\
\text { (texte 7) }\end{array}$ \\
\hline Novices & & & 3 & & & 6 & \\
\hline Débutants & & & 3 & & & 6 & 1 \\
\hline Chevronnés9 & & 1 & 2 & 1 & & 1 & \\
\hline
\end{tabular}

Les groupes des novices et des débutants ont une nette préférence pour les textes concrets 6 et 3 tandis que les interprètes chevronnés semblent partagés, ce qui pourrait indiquer que ces passages n'étaient pas de nature trop difficile à leurs yeux. Les deux interprètes chevronnés qui ont dit que les textes 2 et 4 étaient les plus faciles ont 
une longue expérience de travail pour des institutions gouvernementales et financières (donnée provenant de l'entretien). La familiarité et la connaissance du sujet semblent jouer un rôle important.

\section{COMPARAISON DE TROIS TEXTES : \\ 1 (PYRÉNÉES), 3 (ARCHBISHOP) ET 4 (RUSSIAN ECONOMY)}

En me fondant sur l'hypothèse de départ selon laquelle les textes concrets seraient les plus faciles à restituer et vice versa pour les textes abstraits, $\mathrm{j}$ 'ai sélectionné trois textes qui, selon moi, devaient être les textes le plus facile, le plus difficile et un texte intermédiaire. Les réponses des répondants aux questions concernant les textes le plus facile et le plus difficile correspondaient au choix que j'avais fait. Pour vérifier, j'ai demandé de comparer ces trois textes. Alors que tous les novices (sauf 1 ) et les débutants ainsi que 3 des chevronnés préféraient le texte 3 , les deux interprètes chevronnés qui ont indiqué que le texte 4 était le plus simple à reproduire travaillaient tous deux pour des institutions financières internationales. Le débutant qui choisit le texte 1 vient de la région de Pau et connaissait bien la coutume décrite, ce qui suggère que la connaissance du sujet contribue grandement à la reproduction du texte.

Quel passage est le plus facile à restituer?

TABLEAU 3

Comparaison entre les textes 1 (Pyrénées), 3 (Archbishop) et 4 (Russian Economy)

\begin{tabular}{|l|c|c|c|}
\hline & $\begin{array}{c}\text { Pyrénées } \\
\text { (texte 1) }\end{array}$ & $\begin{array}{c}\text { Archbishop } \\
\text { (texte 3) }\end{array}$ & $\begin{array}{c}\text { Russian Economy } \\
\text { (texte 4) }\end{array}$ \\
\hline Novices & & 8 & 1 \\
\hline Débutants & 1 & 8 & 2 \\
\hline Chevronnés $^{10}$ & & 3 & 1 \\
\hline
\end{tabular}

Une forte majorité dans les trois catégories préfère le texte 3 (Archbishop) bien qu'il soit beaucoup plus long que les deux autres. Ceci est-il dû au fait que ce texte est plus concret et se prête davantage à l'imagerie et à la représentation mentale?

\section{CORRÉLATION ENTRE L'UTILITÉ PERÇUE DE L'IMAGERIE, LE NOMBRE D’ÉLÉMENTS CONCEPTUELS ET LA QUALITÉ DE LA REPRODUCTION DE L'EXTRAIT}

Après avoir établi que les textes concrets étaient plus faciles à restituer, j'ai voulu établir un lien entre cette facilité et l'utilité des images formées telle que les répondants l'ont perçue. Sur la base des réponses exprimées au tableau 4, j'ai retenu les deux textes extrêmes, celui pour lequel l'imagerie avait été la plus utile (texte 3 : Archbishop) et la moins utile (texte 4: Russian Economy). 
TABLEAU 4

L'imagerie a-t-elle été utile lors de la reproduction des extraits?

\begin{tabular}{|c|c|c|c|c|c|c|c|c|c|c|c|c|c|c|c|c|c|c|c|c|c|c|c|c|c|c|c|c|c|c|c|}
\hline Utilité de l'imagerie & & $\mathrm{yr}$ & & & & & $\mathrm{Cl}$ & & & & & & & & & & & & & & & & & assi & & & & & & & \\
\hline Niveau d'imagerie & 1 & 2 & 3 & & 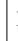 & 2 & 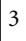 & & 5 & 1 & & 7 & 5 & 1 & 2 & 3 & 4 & 51 & 2 & 3 & 4 & 5 & 1 & 2 & & 5 & 1 & 2 & & & 5 \\
\hline Novices (9) & 1 & 2 & 3 & & . & - & 3 & 5 & 1 & 4 & 2 & 1 & - & - & - & 2 & 6 & - & - & 4 & 3 & 2 & 4 & 3 & & & 1 & 3 & J & & - \\
\hline Débutants (10) & 2 & 5 & 1 & & 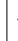 & 1 & 2 & 3 & 4 & 4. & 3 & - & - & - & 2 & 2 & 2 & $4-$ & - & 3 & 2 & 5 & 5 & 4 & & 1 & 4 & & 1 & 2 & - \\
\hline Chevronnés $(7)^{11}$ & - & 2 & 2 & & & - & - & 1 & & 1 & 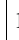 & & & - & - & 1 & & - & - & & & & 2 & 1 & & 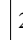 & 1 & & & 1 & 2 \\
\hline
\end{tabular}

1: Extrêmement utile

2: Très utile

3: Utile

4: Pas très utile

5: Pas utile du tout (comprend également les exemples pour lesquels aucune image n’a été formée).

Ce tableau indique le nombre de personnes ayant choisi une des options de 1 à 5. Dans les trois groupes, les textes pour lesquels l'imagerie a été moins utile au dire des répondants sont les textes 2,4 et 5, qui sont également les textes les plus abstraits. Par contre, l'imagerie s'est avérée une aide précieuse pour les textes concrets 3, 6 et 7, quelle que soit leur longueur.

\section{ANALYSE QUALITATIVE}

Jusqu'ici, j'ai montré comment les répondants avaient " perçu» les différents extraits. J'ai voulu établir un lien entre cette perception et la qualité des restitutions. Pour cela, j'ai comparé les textes réputés le plus facile et le plus difficile.

Une analyse sémantique de ces textes montre que le texte 3 (courbe noire - 185 mots) contient 29 concepts et le texte 4 (courbe grise - 74 mots) en comporte 12 . J'ai ensuite compté le nombre d'erreurs sémantiques commises lors de la restitution de chacun de ces textes (sans tenir compte des erreurs grammaticales et terminologiques). Le tableau 5 ci-dessous présente le nombre d'erreurs commises par les novices, les débutants et les interprètes expérimentés sur un total de 29 concepts pour le texte 3 (Archbishop) et 12 pour le texte 4 (Russian Economy) (cf. l'appendices 3).

TABleau 5

Nombre d'erreurs conceptuelles commises pour les textes 3 et 4

\begin{tabular}{|l|c|c|c|c|c|c|c|c|c|c|c|c|c|c|c|c|c|c|c|c|c|c|c|c|c|c|}
\hline & \multicolumn{1}{|c|}{$\begin{array}{c}\text { Novices } \\
\text { (de } 1 \text { à 9) }\end{array}$} & \multicolumn{1}{c|}{$\begin{array}{c}\text { Expérimentés } \\
\text { (de } 19 \text { à 26 }\end{array}$} \\
\hline Archevêque & 5 & 7 & 3 & 3 & 2 & 12 & 6 & 3 & 3 & 2 & 8 & 9 & 7 & 2 & 3 & 6 & 3 & 16 & 29 & 7 & 4 & 14 & 10 & 8 & 14 & 29 \\
\hline Économie russe & 5 & 11 & 1 & 2 & 4 & 5 & 4 & 0 & 7 & 4 & 6 & 6 & 6 & 12 & 2 & 6 & 6 & 12 & 3 & 3 & 4 & 5 & 10 & 6 & 7 & 12 \\
\hline
\end{tabular}

Nous avons ensuite calculé pour chaque texte et chaque répondant le pourcentage du nombre absolu d'erreurs commises afin de pouvoir comparer les deux textes (cf. les tableaux). 
TABLeau 6

Pourcentage d'erreurs conceptuelles commises pour les textes 3 et 4

\begin{tabular}{|c|c|c|c|c|c|c|c|c|c|c|c|c|c|c|c|c|c|c|c|c|c|c|c|c|c|}
\hline \multirow{3}{*}{ Archevêque } & \multicolumn{9}{|c|}{$\begin{array}{l}\text { Novices } \\
\text { (de } 1 \text { à 9) }\end{array}$} & \multicolumn{10}{|c|}{$\begin{array}{l}\text { Débutants } \\
\text { (de } 10 \text { à 19) }\end{array}$} & \multicolumn{6}{|c|}{$\begin{array}{l}\text { Expérimentés } \\
\text { (de } 19 \text { à } 26^{*} \text { ) }\end{array}$} \\
\hline & 17 & 24. & 10. & 10. & 6.9 & & 20. & 10. & 10. & 6.9 & 27. & 31 & 24. 6 & 6.9 & & 20. & 10. 5 & 55. & 10 & 24. & 13. & & 34.2 & & 48. 100 \\
\hline & .2 & 1 & 3 & 3 & & 4 & 7 & 3 & 3 & & 6 & & 1 & & 3 & 7 & 3 & 2 & 0 & 1 & 8 & 3 & 5 & 6 & 3 \\
\hline Économie russe & $\begin{array}{r}41 \\
6\end{array}$ & $\begin{array}{c}91 . \\
7\end{array}$ & 8.3 & $\begin{array}{c}16 . \\
7\end{array}$ & $\begin{array}{c}33 . \\
3\end{array}$ & $\begin{array}{c}41 . \\
7\end{array}$ & $\begin{array}{c}33 . \\
3\end{array}$ & 0 & $\begin{array}{c}58 . \\
3\end{array}$ & $\begin{array}{c}33 . \\
3\end{array}$ & 50 & 50 & 50 & $\begin{array}{c}10 \\
0\end{array}$ & $\begin{array}{c}16 . \\
7\end{array}$ & 50 & \begin{tabular}{l|l}
50 & 1
\end{tabular} & $\begin{array}{c}10 \\
0\end{array}$ & 25 & 25 & $\begin{array}{c}33 . \\
3\end{array}$ & $\begin{array}{c}41 . \\
7\end{array}$ & $\begin{array}{c}83 . \\
3\end{array}$ & 505 & $\begin{array}{c}58 . \\
3\end{array} 100$ \\
\hline
\end{tabular}

Attention: les résultats montrant 100\% d'erreurs correspondent aux répondants qui n'ont pas pu ou qui n'ont pas voulu faire l'exercice (c'est le cas du dernier expert qui ne figure pas sur le graphique).

GRAPHIQUE 1

Analyse qualitative relative de deux textes

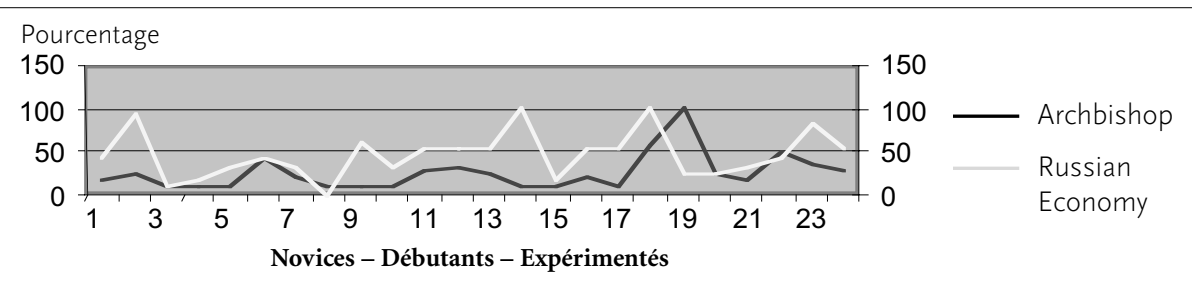

L'analyse qualitative en termes relatifs de ces deux textes indique que:

- Le pourcentage d'erreurs dans le texte sur l'économie russe (courbe grise) est pratiquement toujours supérieur à celui du texte sur l'archevêque (courbe noire). On s'attendrait à ce que ce soit l'inverse vu la longueur respective des textes (74 mots contre 185 mots) et le nombre de concepts (12 contre 29). Le texte sur l'archevêque, plus concret et plus imagé, est mieux rendu que le texte sur l'économie russe.

- Il y a peu de différences entre les trois groupes, ce qui semble nier une hypothèse de départ que les interprètes ayant plus d'expérience devraient avoir moins de difficultés par rapport aux débutants.

- Le meilleur score est obtenu par la répondante \# 8 dont le passage sur l'économie russe est parfaitement restitué. Il est intéressant de remarquer que cette répondante est russe. Sa connaissance ou son intérêt pour le sujet ont-ils joué un rôle?

- Le deuxième pourcentage d'erreurs le plus faible pour l'ensemble des deux textes correspond à la répondante \#3 dont la mémoire est bien entraînée (par sa formation dans le système d'éducation coréen).

- L'interprète probablement le plus expérimenté (\#20 - interprète permanent avec plus de vingt-cinq ans d'expérience) est celui qui a le meilleur résultat des groupes des débutants et des expérimentés. Cette personne est également formateur d'interprètes. Cela a-t-il joué un rôle?

- Les meilleurs résultats sont obtenus dans le groupe des novices (\#3 et 8).

\section{REMARQUES DIVERSES}

Cette étude est limitée par un certain nombre de facteurs dont l'un des principaux est certainement le fait que les renseignements fournis dans les questionnaires par les répondants sont des impressions personnelles qu'ils ont eues à un moment particulier. 
Les résultats ne sont donc utiles qu'à titre indicatif. Rappelons également avec Robert Shepard (1978: 86) que «la raison pour laquelle une image mentale est tellement difficile à étudier de façon empirique ou à expliquer sur le plan conceptuel est qu'elle est un phénomène interne». Nous ne prétendons pas savoir ce qui se passe dans la tête de chaque répondant au moment de l'écoute et de la reproduction des divers messages proposés. C'est pourquoi nous ne disposons que des commentaires plus ou moins précis ou succincts du questionnaire proposé aux répondants et de l'entretien qui a suivi. Même lors d'un «bon» debriefing, comment savoir si les répondants se rappellent toujours de façon très précise les sensations et les impressions qu'ils ont ressenties en entendant le message la première fois? Comment savoir aussi si chacun a fait l'exercice au mieux de ses compétences?

Lors de cette entrevue, trois interprètes chevronnés nous ont surpris de manières différentes: deux disaient travailler uniquement sur la base de mots et non à partir de concepts et le troisième n'établissait, selon lui, aucune relation avec des connaissances préalables lors de l'exercice. Ces remarques reflètent-elles plutôt une approche plus pratique que théorique de la profession et doivent-elles être prises à la lettre? L'entretien après l'exercice avec ces trois répondants montrait que tous les trois avaient une nette préférence pour l'aspect pratique de la profession. Il faut aussi savoir que ce genre de test dépend de la bonne volonté des répondants.

\section{CONCLUSIONS}

L'analyse des restitutions orales de sept passages de longueur, de niveaux d'abstraction et de difficulté différents, données par des interprètes ayant des niveaux d'expérience différents permet de montrer, dans l'ensemble, une nette préférence pour les textes concrets par opposition aux textes abstraits. L'ensemble des répondants indique que l'imagerie a été plus utile pour la restitution des textes concrets, qu'ils estiment également comme étant plus faciles à restituer. L'analyse qualitative du texte le plus concret et du texte le plus abstrait montre que moins d'erreurs sont commises par l'ensemble des répondants lors de la restitution du texte concret, pour lequel tous les répondants disent avoir formé davantage de représentations mentales-images. Il faut toutefois ajouter que d'autres outils utiles sont la connaissance du sujet et la mémoire.

Une des hypothèses de départ selon laquelle il y aurait probablement des différences marquées entre les novices et les interprètes chevronnés quant à la capacité d'utiliser les représentations mentales pour restituer les divers types de textes n'est pas confirmée. Les résultats semblent indiquer en fait que cette capacité ne dépend pas uniquement de l'expérience professionnelle des répondants, mais qu'elle est également liée à d'autres facteurs: la mémoire et la connaissance du sujet. En effet, les meilleurs résultats des deux segments analysés correspondent à des novices dont la mémoire était bien entraînée dans un cas et qui connaissait très bien le sujet dans l'autre. L'importance de la connaissance est confirmée aussi par le fait que les deux textes économiques étaient les mieux rendus par des interprètes qui ont plusieurs années d'expérience dans des organisations économiques. Si la connaissance du sujet est un facteur influençant la qualité de la restitution, il est normal que les personnes plus expérimentées, dans l'ensemble, aient de meilleurs résultats que celles qui n'avaient que des connaissances partielles sur les sujets proposés. Cette différence sera certainement plus marquée pour des sujets beaucoup plus difficiles. La représentation 
mentale, les connaissances du sujet et la mémoire sont trois éléments complémentaires qui contribuent à une meilleure restitution.

L'imagerie, qui est mise en œuvre davantage dans les textes concrets que dans les textes abstraits, semble donc être un outil très utile; elle contribue à une meilleure compréhension et restitution des passages. Lorsque nous analysons aussi bien la représentation mentale que les connaissances sur le sujet et la mémoire des répondants, nous voyons que la difficulté est toujours de savoir exactement ce qui se passe dans la tête de quelqu'un, car nous avons des réalités et des mémoires différentes.

Enfin, un autre facteur dont il faut tenir compte pour comprendre cette étude est qu'elle n'a pas été réalisée de façon aléatoire et porte sur un nombre relativement restreint de 26 personnes (pour le test final). Aussi les observations faites ne peuvent être considérées comme des «conclusions» qui pourraient être généralisées ou s'appliquer à un autre groupe, mais comme de simples indications de ce qui se passe au moment de la compréhension et de la rétention-restitution d'un message. Elles sont à ce titre autant d'encouragements à poursuivre l'étude sur une base plus large.

\section{NOTES}

1. Michel Denis (1989), citant Frandsen et Holder (1969), conclut que face à la résolution de problèmes de logique spatiale, «les sujets qui se représentent les données du problème sous forme d'images visuelles ont $[\ldots]$ de meilleures performances que les sujets de la condition contrôle». Ceci indique que l'utilisation de l'imagerie peut ainsi être considérée comme une stratégie visant à optimiser les conditions d'élaboration de la représentation par le sujet des données du problème.

2. Le Ny, 1982, p. 169: «Pour comprendre une phrase, un texte, un discours, une personne doit créer une représentation sémantique de l'information qui lui est donnée. Ensuite, elle doit pouvoir élaborer une "seconde lecture mentale" de cette représentation soit pour en clarifier le contenu, comme par exemple dans le cas d'un exercice de rappel de l'information, soit pour répondre à des questions de compréhension.» "To understand a sentence, a text, a speech, the subject has to form a semantic representation from information given to him. Then he must be able to make a "mental second reading" of this representation so as to either make clear its content, in a recall task for example, or to answer questions of comprehension."

3. Shepard, 1978, p. 84: (Einstein disait qu'il "ne pensait rarement en termes de mots (Wertheimer, 1945, p. 184). "Il explique que son "abilité particulière" n’était pas les mathématiques mais bien de "visualiser... les effets, les conséquences et les possibilities" (Holton, 1972, p. 110). Il ne pouvait exprimer par des mots et des symboles mathématiques ses découvertes quant à la nature de l'espace et du temps, disait-il, tant qu'il n'avait pas élaboré une conceptualisation de la situation physique grâce à des "images plus ou moins claires qui peuvent être reproduites et combinées de façon "volontaire". (Hadamard, 1945, Annexe 2). "Einstein stated that "he very rarely thought in terms of words at all (Wertheimer, 1945, p. 184). Indeed, he explained that his "particular ability" did not lie in mathematical calculation but rather in "visualizing.... effects, consequences and possibilities" (Holton, 1972, p.110) He could not even undertake the difficult business of finding words and mathematical symbols to communicate his new insights into the nature of space and time, he said, until he had already worked out his conceptualization of the physical situation by means of "more or less clear images which can be "voluntarily" reproduced and combined". (Hadamard, 1945, Appendix 2).

4. Une rare exception est l'ouvrage de R. Setton paru en 1999: Simultaneous Interpretation. A cognitivepragmatic analysis.

5. La représentation verbale ne consiste pas simplement à exprimer nos pensées par des mots, de même que la communication écrite va plus loin que de simplement écrire nos pensées (car elles restent là où elles ont toujours été, c'est-à-dire dans notre cerveau). Il n'y a pas de communication parfaite (Sperber et Wilson, 1986). "Verbal communication is not merely putting our thoughts in terms of words, the same way that written communication is not just writing down our thoughts ("as for our thoughts, they remain where there always were, inside our brain"), there is no perfect communication." 
6. Extrait de Ida Kurcz, Mental Representation of Political Categories: "Representation is usually treated as a mental state or mental process referring to what is represented. The symbolic nature of representations is emphasized. In other words, representations are not copies or reflections of reality. Their function is to amass knowledge and make use of it."

7. Michel Denis (1983) ayant conclu que les résultats étaient meilleurs lorsqu'une consigne d'activation de l'imagerie est donnée aux participants, il était préférable de mesurer le phénomène sans cette activation. On peut supposer que les résultats seraient encore meilleurs si l'on demandait à chaque participant d'essayer d'imaginer au mieux la situation présentée.

8. Deux personnes n'ont pas répondu à cette question.

9. Ibid.

10. Ibid.

11. Ces deux personnes n'ont pas formé d'image, mais disent travailler sur la base de mots, non de concepts.

\section{RÉFÉRENCES}

Adelson, B. (1985) : “Comparing natural and abstract concepts. A case study for computer science." Cognitive Science(9): 417-430.

Anderson, J.-R. (1983): The Architecture of Cognition. Cambridge, Mass, Harvard University Press.

Barsalou, L.-W. et B.-H. Ross (1986): “The role of automatic and strategic processing in sensitivity to superordinate and property frequency.” Journal of Experimental Psychology: Learning, Memory and Cognition 12:116-134.

Bell, R. (1991): Theory and Practice, Longman.

Camus, J.-F. (1996): La psychologie cognitive de l'attention. Paris, Masson et Armand Colin.

Cordier, F. (1993) : Les représentations cognitives privilégiées. Lille, Presses Universitaires de Lille.

Cummins, R. (1988): Meaning and Mental Representation. Cambridge (Mass.) and London (England), The MIT Press-A Bradford Book.

Denis, M. (1989): Image et cognition. Paris, Presses universitaires de France.

_ (1993) : Pour les représentations. Modèles et concepts pour la science cognitive: hommage à J.-F. Le Ny. M. D. G. Sabah. Grenoble, PUG. 1: 95-106.

Denis, M. et S. GÉRARd (1993): Modèles et concepts pour la science cognitive: hommage à J.-F. Le Ny. Grenoble, PUG.

Ehrlich, S. (1982): Construction of Text Representation in Semantic Memory. Language and Comprehension. J.-F. Le Ny, Kintsch, W. Amsterdam, New York, Oxford, North-Holland Publishing Company. 9: 169-178

Francois, J. et Guy Denière (1997): Sémantique linguistique et psychologie cognitive- Aspects théoriques et expérimentaux. Grenoble, Presses universitaires de Grenoble.

Greene, J. (1986). Language Understanding: A Cognitive Approach. Milton Keynes, England, The open University.

Horowitz, M. (1983): Image Formation and Psychotherapy. New York and London, Jason Aronson, Inc.

Jackendoff, R. (1992): Languages of the Mind: Essays on Mental Representation. Cambridge, Massachusetts London, England, The MIT Press.

- (1993): Patterns in the Mind: Language and Human Nature. New York, London, Toronto, Sydney, Tokyo, Singapore, Harvester Wheatsheaf.

Johnson-Laird, P. N. (1983): Mental Models. Cambridge, Massachusetts, Harvard University Press.

Kaufmann, G. (1985) : “A theory of symbolic representation in problem solving." Journal of Mental Imagery 9(2): 51-69.

Kosslyn, S. M. (1983): Ghosts in the Mind's Machine: Creating and Using Images in the Brain. New York, W.W. Norton.

- (1984). Mental representation. Tutorials in Learning and Memory: Essays in Honor of Gordon Bower. J. R. A. a. S.M.Kosslyn. San Francisco, W.H. Freeman : 91-117. 
Kurcz, I. (1993) : Mental representation of political categories. Modèles et concepts pour la science cognitive, hommage à J-F. Le Ny. PUG. Grenoble, Presses universitaires de Grenoble. 1: 65-78. Mental representation of political terms such as "Peace", "Freedom", "Justice".

Lakoff, G. et J. Mark (1985): Les métaphores dans la vie quotidienne, Les Éditions de Minuit.

LE Ny, J.-F. (1985) : Comment (se) représenter les représentations. Les représentations. S. E. (Ed.). $30: 231-238$.

— (1990): "Les modèles de la psychologie cognitive et la psycho-pathologie." Document 84 du CEPCO, Université Paris-Sud.

Le Ny, J.-F. et K. Walter Ed. (1982): Language and Comprehension. Advances in Psychology. Amsterdam, New York, Oxford, North-Holland Publishing Company.

Luger, G. F. Carl Stern, P. J., Newman J. E., R. Yeo (1994): Cognitive Science: The Science of Intelligent Systems. San Diego, New York, Boston, London, Sydney, Tokyo, Toronto, Academic Press.

Marks, D. F. (1973) : "Visual imagery differences in the recall of pictures." British Journal of Psychology 64: 17-24.

Millet, L. (1972): Perception, imagination, mémoire. Paris, Masson et Cie.

Murphy, G.-L. et J.-C. Wright (1984) : "Changes in conceptual structure with expertise: differences between real-world experts and novices." Journal of Experimental Psychology: Learning, Memory and Cognition (10): 144-155.

RichaRD, J.-F. (1990): Les activités mentales: comprendre, raisonner, trouver des solutions. Paris, Armand Colin.

SABAн, G. (1993): Vers une conscience artificielle? Modèles et concepts pour la science cognitive, hommage à J.-F. Le Ny. M. D. G. Sabah. Grenoble, PUG. 1: 207-222.

Seleskovitch, D. (1984): Interpréter pour traduire. Didier Érudition.

Setton, R (1999): Simultaneous Interpretation. A Cognitive-Pragmatic Analysis. Benjamins Translation Library, 28.

Shepard, R. N. (1978): The Mental Image. Introductory Readings for Cognitive Psychology. R. P. Honeck. Guilford, CT, The Dushkin Publishing Group: 83-97.

Sperber, D. et W. Deirdre (1986) : Relevance: Communication and Cognition. Oxford, UK, Basil Blackwell Ltd.

\section{APPENDICES}

\section{APPENDICE 1}

\section{Description des textes}

\begin{tabular}{|c|c|}
\hline Extraits & Commentaires \\
\hline $\begin{array}{l}\text { Extrait \# } 1 \\
\text { Bruce Böglin (from BBC: At the shoulder of } \\
\text { History) - Cassette Tape } \\
\text { I was born in the Pyrenees, near Pau and you } \\
\text { know... Henri IV of France was born in Pau. He } \\
\text { was christened with a cup of local red wine and } \\
\text { garlic that was rubbed on his lips to make quite } \\
\text { sure that he had the spirit of the region } \\
\text { engrained in him. And I grew up very much in } \\
\text { that kind of atmosphere. I used to speak patois, } \\
\text { the patois of the Pyrenees when I was a kid. I was } \\
\text { about four when I arrived there. It didn't mean } \\
\text { that it shut out the English side because that was } \\
\text { fine for lunches but when it came to tea time, tea } \\
\text { was more or less a religion at home... }\end{array}$ & $\begin{array}{l}\text { Durée : } 32 \text { secondes }-119 \text { mots. } \\
\text { Choix du texte: cette entrevue donnée à la } \\
\text { BBC par un interprète professionnel, Bruce } \\
\text { Böglin, est anecdotique. Elle fait intervenir } \\
\text { plusieurs personnes, qui ont des éléments en } \\
\text { commun (nés à Pau): le narrateur, Bruce, } \\
\text { parle de son expérience en tant qu'interprète } \\
\text { et de Henri IV, roi de France. Cet extrait fait } \\
\text { appel à des connaissances culturelles (Pau, les } \\
\text { Pyrénées, le patois, la tradition décrite du } \\
\text { baptême, la tradition du "tea-time"). }\end{array}$ \\
\hline
\end{tabular}


Extrait \# 2 Ms. H. Clinton: Meeting of The "Society for Research in Child Development", Washington DC, April 3, 1997 — Video Tape ... I have had a chance to look through the book that has been produced for this biennial conference and I know you haven't been in Washington DC since I believe the 1930s, but given the number of topics and presenters it seems like you are more than making up for the fact that you haven't been here for some time. The range of topics that you are addressing and the research that you are discussing touches on every aspects of children and families and the environment in which they grow and develop...

Extrait \# 3 Archbishop of Los Angeles The archbishop of Los Angeles gave his blessing to about one thousand animals during a ceremony which took place in a major football stadium of LA. I attended the meeting because my animal was very weak. He is two years now, which is not too much for a dog, but he has never been very strong. That is why I had decided to have him blessed. They were many animals in front of us but I remember a black and white cow, with a very nice straw hat with flowers, which was in front of the two children in front of me. The little boy was carrying a parrot in a cage and his sister was following him with a small, gray monkey. There was a lot of noise in the stadium as you can image. When it was our turn, I was very proud of my bulldog and he must have behaved really well because the archbishop patted him twice on the head after the blessing. Today I am proud to tell you he is in very good health. The blessing must have worked.

Extrait \# 4 Russian Economy

Good news for the Russian economy. The consumer price index for July has come down 0.2 per cent. This is the result of the fall in the price of fruit and vegetable at the beginning of the summer. As a result, it is hoped that the Russian inflation rate will be well within the 30 per cent goal imposed by the IMF. This is surely the first sign of the long expected economic recovery.

Extrait \# 5 South-East Asia (54 words) Currency devaluations in Southeast Asia have eroded Pakistan's competitive advantage.

Therefore, the State Bank of Pakistan made the decision to devalue its rupee by 8 per cent against the dollar. However, this move only meets one of several conditions set by the IMF and will not immediately lead to approval of an aid package. (Jeanne Lee- GSTI French 1999)
Durée: 30 secondes - 93 mots.

Choix du texte: passage relativement confus. Le sujet est général et fait appel à des connaissances antérieures quant à l'histoire de cet organisme. Toutefois, il fait référence à des éléments relativement concrèts (livre, conférence, Washington DC, l'enfant, la famille). Ce sujet permet difficilement une quelconque anticipation de la part de l'interprète et est souvent rendu au mot à mot (many of you have never been to Washington DC... She has read a book on the Society of Children).

Durée: 1 minute - 185 mots.

Choix du texte: ce passage est très concret; il fait intervenir une représentation spatiale (qui est devant qui) en plus de la représentation conceptuelle. Sujet peu banal, difficile à anticiper, qui exige beaucoup d'attention et de concentration vu la quantité de détails visuels fournis, ce qui ajoute à la difficulté de l'exercice. Malgré sa longueur relative, c'est le passage qui a été le mieux rendu dans l'ensemble même si dans certains cas ce n'est pas la vache mais une personne qui porte le chapeau. («Person who had a straw hat»)

Durée: 27 secondes - 74 mots.

Choix du texte: passage abstrait mais faisant appel à des concepts de la réalité concrète de tous les jours (fruits, légumes) et à une représentation possible d'une tendance à l'aide d'une flèche (représentation symbolique de prise de notes). De plus, les concepts économiques sont expliqués (consumer price index est utilisé plutôt que son sigle CPI. Certaines erreurs sont toutefois apparues: «CPI, the Consumer Product Index...».

Durée: 22 secondes - 54 mots.

Choix du texte: ce passage relativement abstrait et complexe fait référence à des concepts économiques qui exigent certaines connaissances en économie. Plusieurs des éléments présentés sont peu familiers (Asie du Sud-Est, disparition de l'avantage concurrentiel, dévaluation, roupie et peut-être même FMI, enveloppe d'aide...) 
Extrait \# 6 Gassing up a car (71 words)

Select a convenient gas station. Pull into the station, next to an available pump. Turn of the engine, then exit the car. Remove the gas cap, then insert the gas pump. Pull on the gas pump lever until the gas tank has been filled with the desired amount of gas. Remove the gas pump and screw the gas cap back into place. Pay for the gas before leaving the gas station. (Jeanne LeeGSTI French 1999)

\section{Extrait \# 7 Bhutan (242 words)}

So often, an entire place can be boiled down to one sensory memory. The smell of freshly cut grass takes me to the lush green rolling hills of Middle Tennessee where I spent my childhood and to that precious moment in the spring when our mothers declare the weather warm enough for us to run barefoot in the yard. The creamy texture of fresh mozzarella cheese always reminds me of my first trip abroad to Northern Italy where I made the delicious discovery that travelling to a new country offers not just new sights, tastes and sounds but new thoughts as well. Like a shriveled tea leave dropped into hot water, these simple cues unfurl and expand to reveal to reveal full fragrant memories of different stages in life, other places, other cultures. In the tiny Himalayan kingdom of Bhutan, more than anything else it is the silence, the absence of sounds that are so widespread in the rest of the world that I remember. It was the silence that allowed me to listen to the sound of my own breathing for the first time. It is amazing how varied and complicated the movement of air in and out of the lungs can be. Even the sound of wind sweeping dry leaves across the road seemed with tiny details that are lost in the outside world. While biking across Bhutan, I remember being fascinated with the fading echo of a barking dog.
Durée: 27 secondes -71 mots.

Choix du texte: ce passage très visuel, séquentiel, de longeur moyenne, fait appel à des actions que nous effectuons régulièrement et donc à une étroite relation avec la réalité. Il exige une écoute attentive pour éviter le danger d'imposer notre connaissance du sujet (exemple: Replace the gas nozzle on its stand - choose an inexpensive gas station...) ou d'omission d'une étape évidente (next to an available station).

Durée: 1 minute 25 secondes - 242 mots. Choix du texte: ce passage est certes plus long et plus "littéraire". Il fait appel à l'imagination et aux sens. Cette double activation de l'imagination et des impressions sensorielles facilite la compréhension du passage. Le fait qu'il comporte une forte structure interne peut aider ceux qui en prennent conscience. Bien que beaucoup de participants considèrent ce passage comme étant très difficile, peu d'erreurs sont commises. 


\section{APPENDICE 2}

\section{Idées du texte 3}

Idées du texte 3 : Archbishop

Novice 1 Novice 2 Novice 3 Novice 4 Novice 5 Novice 6 Novice 7 Novice 8 Novice 9

\begin{tabular}{|c|c|c|c|c|c|c|c|c|c|}
\hline Archbishop & Ok & Ok & Ok & Ok & Ok & Ok & Ok & Ok & Ok \\
\hline Blessing & $\mathrm{Ok}$ & $\mathrm{Ok}$ & $\mathrm{Ok}$ & $\mathrm{Ok}$ & Ok & Ok & Ok & Ok & Ok \\
\hline Animals & Ok & Ok & Ok & Ok & Ok & Ok & Ok & Ok & Ok \\
\hline football stadium & $\mathrm{Ok}$ & $\mathrm{Ok}$ & $\mathrm{Ok}$ & $\mathrm{Ok}$ & $\mathrm{Ok}$ & $\mathrm{Ok}$ & Ok & $\mathrm{Ok}$ & $\mathrm{Ok}$ \\
\hline I was there & Ok & Ok & Ok & Ok & Ok & Ok & Ok & Ok & Ok \\
\hline my bulldog is weak & Ok & Ok & Ok & Ok & Ok & Ok & Ok & Ok & Ok \\
\hline 2 years is not much & Ok & Ok & Ok & Ok & Ok & Wrong & $\mathrm{Ok}$ & Missing & Ok \\
\hline line-up & Ok & Ok & Ok & Ok & Ok & Ok & Ok & Ok & Ok \\
\hline many animals & Ok & Ok & Ok & Ok & Ok & Ok & $\mathrm{Ok}$ & Ok & Ok \\
\hline black/white cow & Ok & Ok & Ok & Ok & Ok & Ok & Wrong & Ok & Ok \\
\hline straw hat & Ok & Ok & $\mathrm{Ok}$ & Ok & Ok & Ok & Ok & Ok & Ok \\
\hline flowers & Ok & Missing & Missing & Ok & Ok & Ok & Missing & Ok & Ok \\
\hline 2 children & ${ }^{\mathrm{Ok}}$ & $\begin{array}{l}\text { Missing - } \\
\text { young bo }\end{array}$ & ok & $\mathrm{Ok}$ & Ok & Ok & Ok & Ok & Ok \\
\hline boy & Ok & $\mathrm{Ok}$ & $\mathrm{Ok}$ & Ok & Ok & Missing & Ok & Ok & Ok \\
\hline parrot & Ok & $\mathrm{Ok}$ & $\mathrm{Ok}$ & Ok & Ok & Missing & Ok & Ok & Ok- green \\
\hline cage & ${ }^{\mathrm{Ok}}$ & $\begin{array}{l}\text { Wrong - } \\
\text { n shoulde }\end{array}$ & $\mathrm{er}^{\mathrm{Ok}}$ & Ok & Ok & Missing & Missing & Ok & Missing \\
\hline girl & $\begin{array}{l}\text { Ok - } \\
\text { sister }\end{array}$ & Missing & $\begin{array}{l}\text { Ok - } \\
\text { sister }\end{array}$ & $\begin{array}{l}\text { Ok- } \\
\text { sister }\end{array}$ & Ok & Missing & Ok & $\mathrm{Ok}$ & Ok \\
\hline girl following boy & Missing & Missing & Ok & Missing & Missing & Missing & Missing & Missing & g Missing \\
\hline small gray monkey & Ok & Ok & Ok & Ok & Ok & Missing & Missing & Ok & Ok \\
\hline me & Ok & Ok & Ok & Ok & Ok & Ok & Ok & Ok & Ok \\
\hline sequence of line & Missing & Wrong & $\mathrm{Ok}$ & $\mathrm{Ok}$ & $\mathrm{Ok}$ & $\mathrm{Ok}$ & $\mathrm{Ok}$ & Ok & $\mathrm{Ok}$ \\
\hline noise & Missing & Ok & Missing & Missing & Ok & Missing & Missing & Missing & g Missing \\
\hline I am proud & Missing & Missing & $\begin{array}{l}\text { Missing } \\
\text { - happy }\end{array}$ & Missing & Missing & Missing & $\mathrm{Ok}$ & Ok & Ok \\
\hline good behavior of dog & Ok & Ok & Ok & Ok & Ok & Missing & Ok & Ok & Ok \\
\hline double petting & Missing & $\mathrm{Ok}$ & $\mathrm{Ok}$ & Ok & Ok & Missing & Ok & $\mathrm{Ok}$ & Ok \\
\hline dog in good health & Ok & Ok & Ok & Ok & Ok & Ok & Ok & Ok & Ok \\
\hline \multirow[t]{2}{*}{ blessing worked } & Ok & Ok & Ok & Ok & Ok & Ok & Ok & Ok & Ok \\
\hline & $5 / 29$ & $7 / 29$ & $3 / 29$ & $3 / 29$ & $2 / 29$ & $12 / 29$ & $6 / 29$ & $3 / 29$ & $3 / 29$ \\
\hline
\end{tabular}




\begin{tabular}{|c|c|c|c|c|c|c|c|c|c|c|}
\hline $\begin{array}{l}\text { Idées du texte } 3: \\
\text { Archbishop }\end{array}$ & $\begin{array}{c}\text { Débutant } 1 \\
(\# 10)\end{array}$ & $\begin{array}{c}\text { 1Débutant } 2 \\
(\# 11)\end{array}$ & $\begin{array}{l}\text { 2Débutant } 3 \\
\text { (\#12) }\end{array}$ & $\begin{array}{l}\text { 3Débutant } 4 \\
\quad \text { (\#13) }\end{array}$ & $\begin{array}{l}\text { Débutant } 5 \mathrm{I} \\
\quad(\# 14)\end{array}$ & $\begin{array}{l}\text { Débutant } 61 \\
\quad(\# 15)\end{array}$ & $\begin{array}{l}\text { 6Débutant } 7 \\
\text { (\#16) }\end{array}$ & $\begin{array}{l}\text { 7Débutant } 8 \\
\quad \text { (\#17) }\end{array}$ & $\begin{array}{l}\text { 8Débutant 91 } \\
\quad(\# 18)\end{array}$ & $\begin{array}{l}\text { 9Débutant } 1 \\
\text { (\#19) }\end{array}$ \\
\hline Archbishop & Ok & Ok & Ok & Ok & Ok & Ok & Ok & Ok & Ok & $\begin{array}{l}\text { did not } \\
\text { do it }\end{array}$ \\
\hline Blessing & Ok & Ok & Ok & Ok & Ok & Ok & Ok & Ok & Ok & \\
\hline Animals & Ok & Ok & Ok & Ok & Ok & Ok & Ok & Ok & Ok & \\
\hline football stadium & Ok & Ok & Ok & Ok & Ok & $\mathrm{Ok}$ & Ok & Ok & Missing & \\
\hline I was there & Ok & Ok & Ok & Ok & $\mathrm{Ok}$ & $\mathrm{Ok}$ & Ok & Ok & Ok & \\
\hline my bulldog is weak & Ok & Ok & Ok & Ok & Ok & Ok & Ok & Ok & Ok & \\
\hline 2 years is not much & Ok & Missing & Ok & Ok & Ok & Missing & Ok & Ok & Missing & \\
\hline line-up & Ok & Ok & Ok & Wrong & Ok & $\mathrm{Ok}$ & Ok & Ok & Missing & \\
\hline many animals & Ok & Ok & Ok & Missing & Ok & $\mathrm{Ok}$ & Ok & Ok & Missing & \\
\hline black/white cow & Ok & Ok & Wrong & Ok & Ok & $\mathrm{Ok}$ & Ok & Ok & Missing & \\
\hline straw hat & Ok & Ok & Missing & Ok & Ok & Ok & Missing & Ok & Ok & \\
\hline flowers & Ok & Missing & Ok & Missing & Ok & Missing & Missing & Daisy & Missing & \\
\hline 2 children & Ok & Ok & Ok & Ok & Ok & Ok & Ok & Ok & Ok & \\
\hline boy & Ok & Ok & Ok & Ok & Ok & Ok & Ok & Ok & Ok & \\
\hline parrot & Ok & Ok & Wrong & Ok & Ok & Ok & Ok & Ok & Missing & \\
\hline cage & Ok & Missing & Ok & Ok & Ok & Ok & Ok & Ok & Missing & \\
\hline girl & Ok & Ok & Ok & $\begin{array}{l}\text { Ok- } \\
\text { (sister) }\end{array}$ & $\begin{array}{l}\text { Ok - } \\
\text { sister }\end{array}$ & Ok & Ok & Ok & Ok & \\
\hline girl following boy & Missing & Missing & Missing & \multicolumn{3}{|c|}{$\begin{array}{l}\text { Missing Wrong - Missing } \\
\text { next to him }\end{array}$} & Missing & Missing & Missing & \\
\hline small gray monkey & Ok & Missing & Missing & $\mathrm{Ok}$ & Ok & $\mathrm{Ok}$ & Missing & Ok & Missing & \\
\hline me & Ok & Ok & Ok & Ok & Ok & Ok & Ok & Ok & Missing & \\
\hline sequence of line & Ok & Ok & Wrong & Wrong & Wrong & Ok & Ok & Ok & Missing & \\
\hline noise & Ok & Missing & Ok & Missing & Ok & Ok & Missing & Missing & Missing & \\
\hline I am proud & Missing & $\begin{array}{l}\text { Missing- } \\
\text { happy }\end{array}$ & Missing & $\begin{array}{l}\text { Missing- } \\
\text { happy }\end{array}$ & $\mathrm{Ok}$ & Ok & Missing & Missing & Missing & \\
\hline good behavior of dog & Ok & Ok & Ok & Ok & Ok & Ok & Ok & Ok & Missing & \\
\hline double petting & Ok & Ok & Ok & Ok & Ok & Ok & Ok & Ok & Missing & \\
\hline dog in good health & Ok & Ok & Missing & Ok & Ok & Ok & Ok & Ok & Ok & \\
\hline blessing worked & Ok & Missing & Missing & & Ok & $\mathrm{Ok}$ & Ok & Ok & Ok & \\
\hline & $2 / 29$ & $8 / 29$ & $9 / 29$ & $7 / 29$ & $2 / 29$ & $3 / 29$ & $6 / 29$ & $3 / 29$ & $16 / 29$ & $29 / 29$ \\
\hline
\end{tabular}




\begin{tabular}{|c|c|c|c|c|c|c|c|}
\hline $\begin{array}{l}\text { Idées du texte } 3 \text { : } \\
\text { Archbishop }\end{array}$ & $\begin{array}{c}\text { Expert } 1 \\
(\# 20)\end{array}$ & $\begin{array}{c}\text { Expert } 2 \\
(\# 21)\end{array}$ & $\begin{array}{c}\text { Expert } 3 \\
(\# 22)\end{array}$ & $\underset{(\# 23)}{\text { Expert } 4}$ & $\begin{array}{c}\text { Expert } 5 \\
(\# 24)\end{array}$ & $\begin{array}{c}\text { Expert } 6 \\
(\# 25)\end{array}$ & $\begin{array}{c}\text { Expert } 7 \\
(\# 26) \\
\text { did not do } \\
\text { the exercice }\end{array}$ \\
\hline Archbishop & Ok & Ok & Missing & Ok & Ok & Ok & \\
\hline Blessing & Ok & Ok & Ok & Ok & Ok & Ok & \\
\hline Animals & $\begin{array}{l}\text { add } 1000 \\
\text { people }\end{array}$ & Ok & Ok & Ok & Ok & Ok & \\
\hline football stadium & $\mathrm{Ok}$ & Ok & Ok & Missing & Ok & Ok & \\
\hline I was there & Ok & Ok & Ok & Ok & Ok & Ok & \\
\hline my bulldog is weak & Ok & Ok & Ok & Ok & Ok & Ok & \\
\hline 2 years is not much & Ok & Ok & Missing & Ok & Ok & Missing & \\
\hline line-up & Ok & Ok & Ok & Missing & Ok & Missing & \\
\hline many animals & Missing & Missing & Ok & $\mathrm{Ok}$ & Ok & Missing & \\
\hline black/white cow & Ok & Ok & Ok & Ok & Ok & Ok & \\
\hline straw hat & Ok & Ok & Ok & Ok & $\mathrm{Ok}$ & Missing & \\
\hline flowers & Ok & Ok & Missing & Missing & Missing & Missing & \\
\hline 2 children & Ok & Ok & Missing & Ok & Ok & Ok & \\
\hline boy & $\mathrm{Ok}$ & Ok & Missing & $\begin{array}{r}\text { Missing } \\
\text { - child }\end{array}$ & Missing & Missing & \\
\hline parrot & Ok & Ok & Ok & Ok & Ok & Missing & \\
\hline cage & & Ok & Missing & Missing & Missing & Missing & \\
\hline girl & Ok & $\begin{array}{c}\text { Ok } \\
\text { (sister) }\end{array}$ & Missing & $\begin{array}{l}\text { Missing } \\
\text { - child }\end{array}$ & Missing & Missing & \\
\hline girl following boy & Missing & Missing & Missing & Missing & Missing & Missing & \\
\hline small gray monkey & Ok & Ok & Ok & Ok & Ok & Ok & \\
\hline me & Ok & Ok & Ok & Ok & Ok & Ok & \\
\hline sequence of line & Ok & Ok & Missing & Ok & Ok & Wrong & \\
\hline noise & Missing & Ok & Missing & Missing & Missing & Missing & \\
\hline I am proud & Missing & Ok & Missing & $\begin{array}{l}\text { Missing } \\
\text { - happy }\end{array}$ & $\begin{array}{l}\text { Missing } \\
\text { - happy }\end{array}$ & Ok & \\
\hline good behavior of dog & Missing & Ok & Missing & Ok & Missing & Ok & \\
\hline double petting & Missing & Ok & Missing & Missing & Missing & Ok & \\
\hline dog in good health & $\mathrm{Ok}$ & Missing & Ok & $\mathrm{Ok}$ & Ok & Ok & \\
\hline \multirow[t]{2}{*}{ blessing worked } & Ok & Missing & Missing & Ok & Ok & Ok & \\
\hline & $7 / 29$ & $4 / 29$ & $14 / 29$ & $10 / 29$ & $8 / 29$ & $14 / 29$ & $29 / 29$ \\
\hline
\end{tabular}




\section{APPENDICE 3}

\section{Idées du texte 4}

Concepts du texte 4:

Économie russe

Good news

Russian economy

CPI

July

Down . $2 \%$

Due to decrease price

Fruit \& vegetable

Beginning Summer

Hope

Inflation rate $<30 \%$

IMF decision

Sign of recovery
Novice 1 Novice 2 Novice 3 Novice 4 Novice 5 Novice 6 Novice 7 Novice 8 Novice 9

$\begin{array}{llllllll}(\# 1) & (\# 2) & (\# 3) & (\# 4) & (\# 5) & (\# 6) & (\# 7) & (\# 8)\end{array}$

Ok Missing Ok $\quad$ Ok $\quad$ Ok $\quad$ Ok $\quad$ Ok $\quad$ Ok $\quad$ Ok

Ok Ok Ok $\quad$ Ok $\quad$ Ok $\quad$ Ok $\quad$ Ok $\quad$ Ok $\quad$ Ok

Ok Missing $\mathrm{Ok} \quad \mathrm{Ok} \quad \mathrm{Ok} \quad \mathrm{Ok} \quad \mathrm{Ok} \quad \mathrm{Ok}$ Missing

Missing Missing Missing Ok Missing Missing Missing Ok Ok

Wrong Wrong Ok Ok Ok Wrong Ok Ok Missing

Ok Missing $\mathrm{Ok} \quad \mathrm{Ok} \quad \mathrm{Ok} \quad \mathrm{Ok} \quad \mathrm{Ok} \quad \mathrm{Ok} \quad \mathrm{Ok}$

Ok Missing Ok $\quad$ Ok $\quad$ Ok $\quad$ Ok $\quad$ Ok $\quad$ Ok $\quad$ Ok

Ok Missing Missing Ok Ok Missing Missing Ok Wrong

Ok Missing Ok Missing Missing Missing Missing ok Missing

Missing Missing Ok Ok Wrong Ok Missing Ok Missing

Wrong Missing $\mathrm{Ok} \quad \mathrm{Ok} \quad \mathrm{Ok} \quad \mathrm{Ok} \quad \mathrm{Ok} \quad \mathrm{Ok}$ Missing

Wrong Wrong Ok Missing Missing Missing Ok Ok Missing

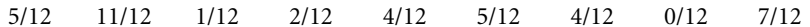

Concepts du texte 4: Débutant 1Débutant 2Débutant 3Débutant 4Débutant 5Débutant 6Débutant 7Débutant 8Débutant 9Débutant 10

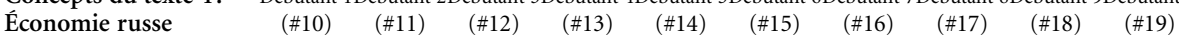

Good news

Ok Ok Ok Missing did not Ok Ok Ok did not $\mathrm{Ok}$

Russian economy

$\begin{array}{cccc}\text { Ok } & \text { Ok } & \text { Ok } & \text { Missing } \\ \text { Ok } & \text { Ok } & \text { Ok } & \text { Ok }\end{array}$ do it do it

CPI

Ok Missing Missing Missing

Ok Ok Ok Ok

July

Ok Ok Ok Ok

$\mathrm{Ok}$

Down $.2 \%$

Ok Missing Ok Ok

Missing Missing Wrong

$\mathrm{Ok}$

Ok Wrong Ok (small

$\mathrm{Ok}$

Due to decrease price

Ok Missing Ok Ok amount)

Fruit \& vegetable

$\begin{array}{llll}\text { Ok Missing } & \mathrm{Ok} & \mathrm{Ok} \\ & & \end{array}$

Ok Wrong Ok

Ok

Beginning Summer

Hope

Missing Missing Missing Missing

Inflation rate $<30 \%$

Wrong Ok Missing Wrong

Ok Ok Missing Ok

Ok Ok Ok

Ok

IMF decision

Sign of recovery

Missing Missing missing Wrong

Ok Ok Missing Missing

Ok Wrong Missing Missing

Ok Missing Wrong Ok

Ok Missing Wrong Ok

\begin{tabular}{lccccccc}
\hline $\begin{array}{l}\text { Concepts du texte 4: } \\
\text { Économie russe }\end{array}$ & $\begin{array}{c}\text { Expert } 1 \\
(\# 20)\end{array}$ & $\begin{array}{c}\text { Expert 2 } \\
(\# 21)\end{array}$ & $\begin{array}{c}\text { Expert 3 } \\
(\# 22)\end{array}$ & $\begin{array}{c}\text { Expert } 4 \\
(\# 23)\end{array}$ & $\begin{array}{c}\text { Expert } 5 \\
(\# 24)\end{array}$ & $\begin{array}{c}\text { Expert 6 } \\
(\# 25)\end{array}$ & $\begin{array}{c}\text { Expert 7 } \\
(\# 26)\end{array}$ \\
Good news & Ok & Ok & Ok & Missing & Ok & Ok & $\begin{array}{c}\text { did not do } \\
\text { the exercise }\end{array}$ \\
Russian economy & Ok & Ok & Ok & Ok & Ok & Ok & \\
CPI & Ok & Ok & Ok & Missing & Ok & Ok \\
July & Missing & Missing & Missing & Missing & Missing & Missing \\
Down .2\% & Wrong & Ok & Wrong & Missing & Ok & Wrong \\
Due to decrease price & Ok & Ok & Ok & Missing & Missing & Wrong & \\
Fruit \& vegetable & Ok & Ok & Ok & Missing & Missing & Ok & Ok \\
Beginning Summer & Ok & Missing & Ok & Missing & Missing & Ok \\
Hope & Ok & Wrong & Missing & Missing & Missing & Missing & \\
Inflation rate $<30 \%$ & Ok & Ok & Ok & Wrong & Ok & Missing & \\
IMF decision & Ok & Ok & Missing & Ok & Ok & Wrong & \\
Sign of recovery & Missing & Missing & Missing & Wrong & Missing & Missing & \\
& 3/12 & $4 / 12$ & $5 / 12$ & $10 / 12$ & $6 / 12$ & $7 / 12$ & $12 / 12$
\end{tabular}




\section{APPENDICE 4}

\section{Instrument}

\section{Recall exercise :}

Please listen carefully to the following excerpt. Your task is to reproduce it as well as you remember as if you were telling someone you has not had the benefit of reading it. Try to include as many details as possible.

\section{Exemple 1}

Bruce Böglin (from BBC: At the shoulder of History) - Cassette Tape

\section{INDIVIDUAL DEBRIEFING}

1) What was the most difficult term in the passage you heard?

2) Was the passage easy to understand?

$$
\begin{array}{ccccc}
1 & 2 & 3 & 4 & 5 \\
\text { Very Easy } & \text { Easy } & \text { Normal } & \text { Difficult } & \text { Very difficult }
\end{array}
$$

3) Was this information easy to recall? Please mark on a scale from 1 to 5

$\begin{array}{ccccc}1 & 2 & 3 & 4 & 5 \\ \text { Very Easy } & \text { Easy } & \text { Normal } & \text { Difficult } & \text { Very difficult }\end{array}$

4) Are you aware of any passage in this excerpt you have misunderstood?

$$
\text { Yes No }
$$

5) Did you use mental representation/image when you were reproducing the message? Yes No

6) What kind of pictures do you remember now?

7) How useful was(ere) the image(s) you formed to reexpress the original message?

$$
\text { Extremely useful Very useful Useful Not very useful Not useful at all }
$$

8) Have you established any connection between what you heard and some previous knowledge?

$$
\text { Yes No }
$$

If Yes, which one (explain)

9) How familiar were you with the topic presented?

$$
12 \quad 3
$$

Extremely familiar Very familiar Familiar Not very familiar Not familiar at all




\section{Dear respondant :}

Thank you for accepting to take part in this short study. The information you will provide will be used in such a way that the confidentiality of the data will be respected and the identity of the respondants protected.

You don't have to identify yourself.

Last Name:

First Name:

Male/ Female

Language Combination:
A. language:
B. language:
C. language:

If your mother tongue is different from English, how long have you studied English?

Have you already worked as an interpreter? Yes No

If yes, was it:

1. Occasionnally

2. Regularly

3. Frequently

4. Very often

5. On a full time basis

Have you been trained as an interpreter? Yes No

If yes, was it: 1. a workshop (one or two days)

2. a short course (up to one week)

3. a seminar (no more than 3 months)

4. a bachelor's program

5. a master's program

If no, for how long have you been working as an interpreter?
less than 1 month
between 1 month and 1 year
between $1-5$ years
between $5-10$ years
more than 10 years

Thank you for your participation! 


\section{GENERAL DEBRIEFING}

1) Which was the most difficult excerpt to understand and why?

Check or circle the excerpt that was most difficult.

Bruce Böglin

H. Clinton

Blessing of the animals

Russian Economy

Currency devaluations in Southeast Asia

How to gas up a car?

Bhutan

2) Which was the easiest excerpt to understand and why?

Check or circle the excerpt that was easiest to understand.

Bruce Böglin

H. Clinton

Blessing of the animals

Russian Economy

Currency devaluations in Southeast Asia

How to gas up a car?

Bhutan

3) Was the passage on the Russian economy easier that the one on Devaluation in Southeast Asia?

Yes No

4) Was the passage on Gassing up a car easier that the one on Bhutan?

Yes No

5) Was the passage on Bhutan easier than the language used by Ms. Clinton?

Yes

No

6) Which of the following passages was easiest to reproduce?
1: Pyrenees
2: Blessing of animals
3: Russian Economy

7) In general, how useful was the mental representation/image when you had to reproduce the message?

1

23

4

Extremely Useful Useful Not very useful Not useful at all I didn't create any representation 